\title{
An interpretation of age-related differences in letter-matching performance
}

\author{
JULIE MAPES LINDHOLM and STANLEY R. PARKINSON \\ Arizona State University, Tempe, Arizona
}

\begin{abstract}
Comparisons were made of the response latencies of old (mean age $=69.2$ years) and young (mean age $=26.8$ years) subjects on simple and choice reaction time (RT) tasks and "physical identity" (PI) and "name identity" (NI) trials of a letter-matching task. Young subjects were faster than old subjects on all tasks, and the absolute difference between groups increased with processing complexity (simple RT < choice RT < PI < NI). However, in support of the hypothesis that aging is associated with a general reduction in processing speed, the relative difference between groups did not vary with task, except for a subset of the NI trials. Response latencies for the NI trials varied with stimulus letter for both age groups, but the magnitude of the letter effect was greater for the elderly. Their latencies were disproportionately long for the more difficult letters. A second experiment showed that NI latency reflected the visual similarity of the letters with respect to the other letters in the stimulus set. It is suggested, therefore, that the NI letter effect resulted from differences in letter identification time. The disproportionately long latencies of the elderly for the visually similar letters are discussed in terms of the hypothesis that aging is associated with an increase in internal noise.
\end{abstract}

The study of individual and age-related differences from an information processing perspective has met with only limited success. Estimates of the parameters of certain models have shown considerable instability or have been inconsistently related to each other and to psychometric measures (e.g., Chiang \& Atkinson, 1976; Hunt, 1978; Hunt \& MacLeod, 1978; Welford, 1977). In view of such results, Hunt (1978) argued that it is important to distinguish between automatic and controlled (attention-demanding) processes. He suggested that stable individual and developmental differences exist in the speed with which the automatic processes are executed, whereas the controlled processes are subject to intraindividual variation in efficiency.

In Hunt's work (Hunt, 1978; Hunt, Frost, \& Lunneborg, 1973; Hunt, Lunneborg, \& Lewis, 1975), performance on the letter-matching task designed by Posner and his associates (Posner \& Mitchell, 1967) has been used as an indicant of automatic processing efficiency. In this task, subjects judge whether two visually presented letters are the same or different. The two letters are identical, different, or physically different with the same name, and subjects are instructed to make a "same" judgment

This research was supported in part by grants from the National Institute on Aging (AGO2130) and the National Institute of Mental Health (MH26870). We thank Peter Killeen, Ernest Lindholm, Clark Presson, and Susan Somerville for helpful comments on an earlier draft of this paper. Requests for reprints should be sent to Julie Lindholm, Department of Psychology, Arizona State University, Tempe, Arizona 85287. on the basis of either physical (PI) or name (NI) identity. The basic finding with this paradigm, regardless of the exact experimental variant, is that "same" response latencies are shorter for PI pairs than for NI pairs. This difference in latency is thought to represent the extra time needed to access letter names in long-term memory.

Hunt and his colleagues have found that the NIPI difference is smaller for students with relatively high verbal ability than for students with relatively low verbal ability (Hunt et al., 1975). Moreover, Hunt (1978) presented data from his own and other laboratories which suggest that the NI-PI difference is sensitive to both developmental level and brain dysfunction. He interpreted these findings as evidence that the efficiency with which overlearned information is accessed is an important locus of individual and developmental differences in cognitive functioning (cf. Keating \& Bobbitt, 1978).

In order to argue that the speed of a particular process has such importance, however, it is necessary to show that discriminating differences in efficiency are specific to or disproportionately present in the process under investigation. If, for example, the mean latencies for the PI and NI trials of a letter-matching task were, respectively, 400 and $500 \mathrm{msec}$ for Group $A$ and 600 and $750 \mathrm{msec}$ for Group B (creating a significant group $\times$ condition interaction), it would not be meaningful to conclude that the two populations can be differentiated on the basis of the efficiency with which they generate letter names. Although the absolute difference between groups, in this example, is greater 
for the NI trials, the relative difference is invariant: The mean latency of Group B is 1.5 times as great as the mean latency of Group A, for both types of matching. Equivalently, although the absolute magnitude of the NI-PI contrast is greater for Group B than for Group A, both groups show a $25 \%$ greater mean latency for the NI trials than for the PI trials. Such a pattern of results would indicate that the populations differ in information processing speed but would provide negative evidence with respect to the hypothesis that population B is characterized by a specific deficiency in the speed of accessing information in long-term store.

The question of the specificity of processing-speed differences is particularly important in research concerned with the cognitive changes that accompany advancing age. Age-related slowness has been repeatedly demonstrated on a wide variety of information processing tasks (e.g., Anders, Fozard, \& Lillyquist, 1972; Botwinick, 1973; Walsh, 1976; Welford, 1977). The implicit assumption in most investigations is that the elderly are characterized by specific and fairly independent processing deficits (e.g., Anders et al., 1972; Welford, 1977). However, Birren $(1965,1974)$ has suggested that there is a general age-related reduction in processing speed, which affects all aspects of cognitive functioning.

More specifically, it is proposed here that the processing speed of an elderly person, $S_{i}$, is some proportion, $P_{i}$, of his or her processing speed as a young adult. The value of $P_{i}$ is assumed to be independent of original speed. If $K_{i}$ is defined as the reciprocal of $P_{i}$, the average processing time of a random sample of elderly subjects would be $\bar{K}$ (the average of the $K_{i}$ values) times as long as the average processing time of a random sample of young subjects (within sampling error), for all information processing tasks.

Given a low and nonvariable error rate, response latency can be taken as a measure of processing time, and the hypothesis that aging is associated with a general reduction in speed of processing can be tested by comparing the response latencies of different age groups for tasks that vary in processing requirements. For statistical analysis, it is important to note that if latencies are related by the factor $K_{i}, \log$ latencies will differ by the constant $\log K_{i}$. Thus, if the reduction-in-speed hypothesis can account for age-related differences in latency, the difference between the means of a group of old and a group of young subjects on a logarithmic scale should equal mean $\log K$, for all tasks. On the other hand, if aging is associated with some specificity of processing-speed reduction or with other deficits, in addition to a general reduction in speed, the relative difference between age groups should vary with processing requirements.

\section{EXPERIMENT 1}

The purpose of Experiment 1 was to examine agerelated differences in letter-matching performance with respect to the hypothesis that aging is associated with a general reduction in processing speed. In order to provide additional and presumably more basic indices of processing speed than that provided by PI latency, "simple" and "choice" latencies were obtained for a subset of the letter-matching pairs: The subjects were instructed to respond as soon as the letters appeared in the simple RT task and on the basis of letter case in the choice RT task. Since case discrimination could be based on either letter size or the presence of curved line segments, we expected the choice RT task to involve less processing than the PI trials of the letter-matching task, providing a continuum of processing complexity: simple $<$ choice $<$ PI $<$ NI.

\section{Method}

Subjects. The elderly subjects were recruited from a Phoenix chapter of the American Association of Retired People (AARP). Remuneration for their participation was made to the AARP chapter. The younger group included both community residents who responded to a newspaper advertisement and college students. The young subjects recruited through the newspaper were paid for their participation; the remaining young subjects participated in order to fulfill a requirement for an introductory psychology course at Arizona State University.

One elderly and one young (paid volunteer) subject were replaced due to aberrant RTs: The elderly subject's RTs were greater than $2 \mathrm{sec}$ on over $25 \%$ of the trials for both letter-matching sessions; the young subject's RTs were greater than $2 \mathrm{sec}$ on only $1 \%$ of the trials for the first letter-matching session but on $19 \%$ of the trials for the second such session. Two additional young subjects (students) were replaced because they performed with very high error rates: One subject made $>25 \%$ errors in the choice RT task and in both sessions of the letter-matching task; the other subject made $>14 \%$ errors in both sessions of the letter-matching task.

The final sample consisted of 16 old subjects (ranging in age from 62 to 77 , with a mean age of 69.2 years) and 16 young subjects (ranging in age from 17 to 41 , with a mean age of 26.8 years). There were 13 females and 3 males in each group. The formal education of the elderly subjects ranged from 8th grade to postbaccalaureate, averaging 12.1 years. The formal education of the young subjects was somewhat higher, averaging 13.8 years. In contrast to this advantage of the young in years of formal education, the elderly had had many more years of informal education in a variety of occupations (e.g., pharmacist, teacher, business owner, radio operator). Most of the younger subjects who were not students had worked for a few years as teachers or secretaries.

Stimull and Apparatus. The stimulus slides were composed of vinyl plastic letters $(1.27-\mathrm{cm}$ Super Stik Helvetica) between two pieces of glass bound by aluminum frames. Pairs of letters from the set $A, H, T$, and $F$ (both upper- and lowercase) were used for all three tasks.

For the simple and choice RT tasks, half of the slides consisted of two uppercase letters and half of two lowercase letters. Each upper- and lowercase letter was paired three times with itself and two times with each of the other three letters of the same case, once in the left and once in the right position, creating a set of 48 slides. For the letter-matching task, these slides 
were supplemented by a parallel set which consisted of the combinations involving one lower- and one uppercase letter (e.g., $\mathrm{tT}, \mathrm{Ht}$ ). Of the resulting set of 96 slides, 24 were composed of PI letter pairs ( 3 lower- and 3 uppercase pairs per letter) and 24 of NI letter pairs (3 with the uppercase form on the left and 3 with the uppercase form on the right for each letter). The remaining 48 slides consisted of letters with different names (DIFF pairs): Each letter was paired with each of the other letters eight times, with case and position completely balanced (e.g., TH, HT, th, ht, Th, Ht, tH, hT).

A Lafayette 41010 automatic projection tachistoscope was used to present the slides on a white background in a darkened room. The shutter sync of the tachistoscope activated a DurwoodBrown clock, which recorded response latency in milliseconds. The intertrial interval was $8 \mathrm{sec}$, and the stimuli were displayed until a button was pressed.

The projected letters were approximately $17.8 \mathrm{~cm}$ high and were viewed from a distance of approximately $1.2 \mathrm{~m}$. The subjects sat in a straight-backed chair with two fingers resting on the response buttons, which were mounted on a platform attached to the right arm of the chair.

Procedure and Design. The subjects were tested individually for three sessions. During the first session, they received the simple RT task followed by the choice RT task. In both tasks, 16 practice trials were followed by two presentations of a random ordering of the 48 stimulus slides, for a total of 96 test trials. The same stimulus sequence was used in both tasks and for all subjects. In the simple RT task, the subjects were instructed to respond with a buttonpress as soon as a letter pair appeared. Both left and right response latencies were obtained for each subject by dividing the 96 trials into four sets of 24 and changing the required response in accord with an ABBA design. Half of the subjects pressed the left button and half pressed the right button during the first 24 trials. In the choice RT task, subjects were instructed to press one button when the two letters were uppercase and the other button when they were lowercase. Response assignment was balanced across subjects.

The letter-matching task was presented during both Session 2 and Session 3. Each session consisted of 16 practice trials followed by 96 test trials. The 96 stimulus slides were divided into two sets of 48, each containing 12 PI, 12 NI, and 24 DIFF pairs. Slides within a set of 48 were sequenced randomly, and the order in which the two sets were presented was balanced across subjects in a group. The subjects were instructed to indicate whether the two stimulus letters shared the same name. Response assignment (left or right button for "same") was balanced across subjects.

Instructions were read at the beginning of each task and repeated, when necessary, during the practice trials. The subjects were encouraged to respond as quickly as possible without making errors.

\section{Results}

Response latencies. The latency data for each subject were divided into subsets as follows: (1) For the simple RT task, left and right responses were considered separately. (2) For the choice RT task, correct response latencies were partitioned on the basis of letter-pair case. (3) For each session of the letter-matching task, correct "same" responses were classified according to trial type (PI or NI) and letter $(\mathrm{A}, \mathrm{H}, \mathrm{T}, \mathrm{F})$, and correct "different" responses were classified according to letter combination (AH, AT, AF, HF, HT, TF). Because the latency distributions tended to be positively skewed, with an occasional very short (presumably anticipatory) or very long latency, a subject's performance on a task subset was represented by his or her median correct response latency.

In a preliminary analysis of variance conducted on the response latency data for the simple RT task, neither the main effect of response assignment (left vs. right) nor the interaction of response assignment and age was significant. Similarly, the effects involving letter case were nonsignificant in an analysis of latencies for the choice RT task.

For an analysis of variance in which the simple and choice RT tasks and the PI and NI trials of the letter-matching tasks were treated as four levels of "processing complexity," the median response latencies (for the task subsets) were averaged to yield one score per subject per complexity level. These scores are presented in Figure 1, averaged over subjects in a group. Inspection of the data reveals that (1) the proposed ordering of response latencies (simple $<$ choice $<$ PI $<$ NI) was obtained for both age groups; (2) latency varied substantially with complexity level; (3) the average latency of the old group was longer than that of the young group for each level of complexity; and (4) the difference between groups increased with processing complexity. In the analysis of these data, the age $[F(1,30)=33.35]$, complexity $[F(3,90)=472.21]$, and age $\times$ complexity $[F(3,90)=29.56]$ effects were all significant at the .001 level. Subsequent analyses revealed that

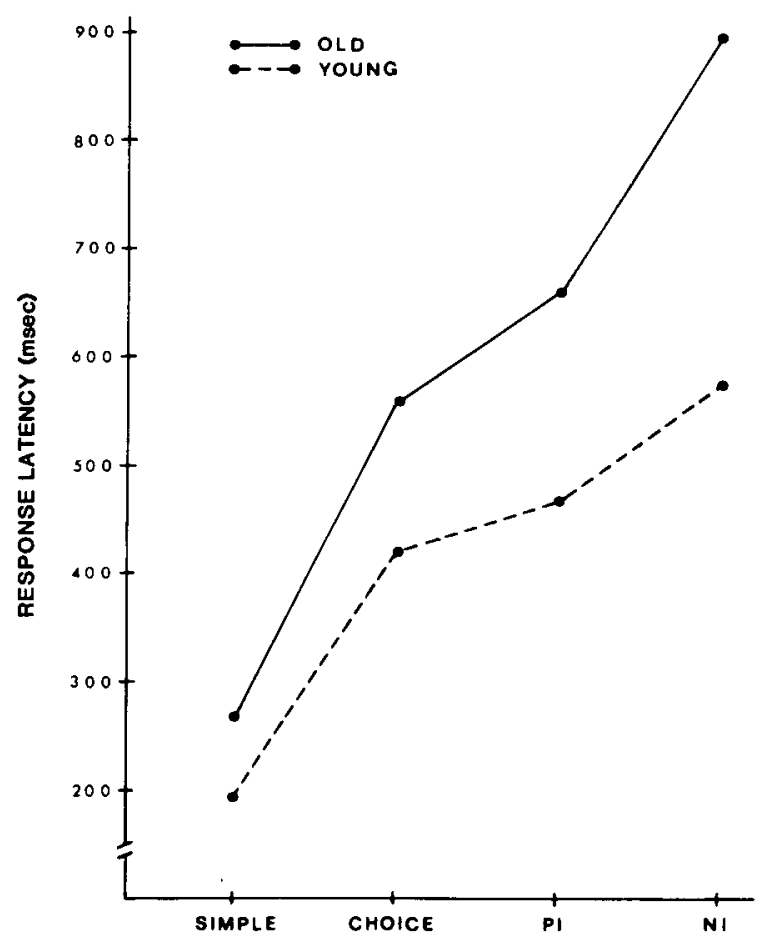

Figure 1. Response latency (milliseconds) as a function of processing complexity for the young and old groups. 
the significance of the interaction was not dependent upon the full range of processing complexity: The age $\times$ complexity interaction was also significant in an analysis restricted to the simple and choice RT tasks $[F(1,30)=6.68, p<.02]$, as well as in an analysis of the simple and choice tasks and the PI trials of the letter-matching task $[F(2,60)=12.61$, $\mathrm{p}<.001]$.

The DIFF and NI trials of the letter-matching task were presupposed to require the same level of processing complexity. This supposition was supported by an analysis of variance of the mean median NI and DIFF latencies for each session: Only the main effects of age and session were significant $[F(1,30)=44.63, p<.001$, and $F(1,30)=15.71$, $\mathrm{p}<.001$, respectively]. Responses to DIFF pairs were slightly, but not significantly, faster than responses to NI pairs (old, mean DIFF $=865 \mathrm{msec}$ and mean $\mathrm{NI}=896 \mathrm{msec}$; young, mean $\mathrm{DIFF}=$ $561 \mathrm{msec}$ and mean $\mathrm{NI}=576 \mathrm{msec}$ ).

As a preliminary test of the uniformity of the agerelated decrement in information processing efficiency, the average latency of the old group was divided by the average latency of the young group for each level of complexity. The resulting quotients were $1.37,1.33,1.41$, and 1.56 for simple, choice, PI, and NI, respectively. Disregarding namematching performance, the similarity of these values is consistent with the hypothesis that aging is associated with a general reduction in processing speed.

In order to evaluate this hypothesis statistically, each subject's scores (mean median latencies) for the simple and choice RT tasks and the PI and NI trials of the letter-matching task were subjected to a logarithmic transform. Because equal ratios on a millisecond scale become equal intervals on a log millisecond scale, the age $\times$ complexity interaction in an analysis of $\log$ milliseconds tests whether old and young were affected disproportionately by task demands. As suggested by the group mean data, the age $\times$ complexity interaction was not significant $(F<1.0)$ in an analysis of the log latencies for the first three levels of processing complexity (simple, choice, PI). The interaction was significant, however, when the log latencies for the NI trials were added to the analysis $[F(3,90)=3.72$, $\mathrm{p}<.02$ ]. Although this latter result is consistent with the hypothesis that there is a specific, agerelated decrease in the speed of accessing letter names, in addition to the general slowing manifest in the less complex tasks, this interpretation was not supported by more detailed analyses.

In order to explore the consistency of the age deficit in name matching efficiency, an analysis of variance was conducted on the logs of the 16 median response latencies (for the combinations of 4 letters, 2 trial types, and 2 sessions) representing a subject's performance on the letter-matching task. These $\log$ latencies are presented in Figure 2, averaged over subjects in a group. The analysis indicated that each of the factors had an overall effect on log latency: Old subjects were slower than young subjects $[F(1,30)=52.57, p<.001]$, NI responses were slower than PI responses $[F(1,30)=319.01$, $\mathrm{p}<.001$ ], Session 1 responses were slower than Session 2 responses $[F(1,30)=10.79, p<.005]$,

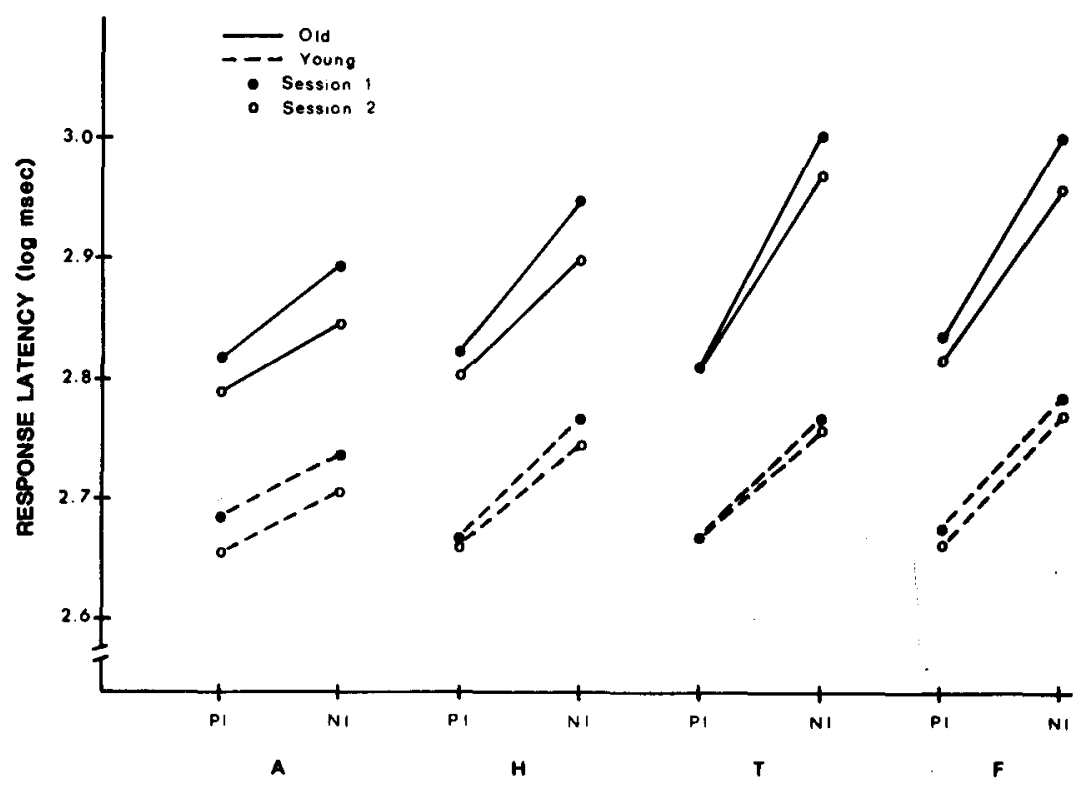

Figure 2. Response latency (log milliseconds) as a function of trial type and letter for the young and old groups in Sessions 1 and 2. 
and response latency varied with stimulus letter $[F(3,90)=21.67, p<.001]$. In addition to these main effects, all but one of the first-order interactions reached significance: The NI-PI difference was greater for old than for young $[F(1,30)=11.93$, $p<.005]$, the letter effect was greater for old than for young $[F(3,90)=4.16, p<.01]$, the NI-PI difference varied with letter $[F(3,90)=21.74, p<.001]$, the NI-PI difference was greater for Session 1 than for Session $2[F(1,30)=7.75, p<.01]$, and the session effect varied with letter $[F(3,90)=3.15$, $p<$ $.05]$. Of greatest importance to our understanding of age differences in letter-matching performance, the age $\times$ trial type $\times$ letter interaction was also significant $[F(3,90)=3.28, p<.025]$ : The effect of letter on the magnitude of the NI-PI difference (i.e., the trial type $\times$ letter interaction) was greater for old than for young subjects. ${ }^{1}$

Analyses of the age $x$ trial type interaction on a letter-by-letter basis revealed that the NI-PI difference for the elderly group was disproportionately large only for the letters $T$ and $F[F(1,30)=16.81$, $\mathrm{p}<.001$, and $\mathrm{F}(1,30)=5.40, \mathrm{p}<.05$, respectively]. Additional subsequent analyses showed that the letter and age $\times$ letter effects were restricted to the NI trials, as neither of these effects was significant in analyses of the latencies and $\log$ latencies for the PI trials. However, while each NI pair contained both an upper- and a lowercase letter, half of the PI pairs consisted of lowercase letters and half of uppercase letters. If the letter effect were restricted to one case, the use of median RTs might have obscured the effect for the PI trials. To test this possibility, mean and mean log latencies for the PI letter pairs were determined for each subject. Since neither the letter nor the age $\times$ letter effects were significant in analyses of these dependent variables, the absence of letter effects for the PI trials was not an artifact of the performance measure.

An analysis of the log median response latencies for the six DIFF letter combinations showed that the latency of a correct "different" response varied significantly with letter combination $[F(5,150)=$ $6.38, \mathrm{p}<.001]$, as well as with age $[\mathrm{F}(1,30)=45.53$, $\mathrm{p}<.001]$ and session $[\mathrm{F}(1,30)=16.23, \mathrm{p}<.001]$. None of the first-order interactions were significant, but there was a significant age $\times$ session $\times$ letter combination effect $[F(5,150)=2.63, p<.05]$. Collapsed over groups, latencies were longest for the TF combination on each of the sessions. Mean latencies for both groups decreased over sessions for each of the letter combinations, with the exception of TF, for which the mean of the elderly group increased slightly. The decreases for the other combinations varied with age such that the ordering of the letter combination means was more consistent across groups on Session 2 than on Session 1. For the young subjects, the magnitude of the letter effect decreased slightly over sessions, whereas for the older subjects it increased. For the TF combination on Session 2, the mean of the elderly group was 1.62 times greater (i.e., a difference of .20898 log units) than the mean of the young group. For the three letter combinations (AT, AF, and TH) which resulted in the shortest latencies, the elderly group's means averaged 1.49 times greater than the young group's means. These values are to be compared with the old to young ratios for NI on Session 2 of 1.58:1 for pairs containing either letter $T$ or letter $F$ and 1.40:1 for pairs containing letter $\mathbf{A}$.

Errors. The overall error rates were low for both the choice and letter-matching tasks. In the choice RT task, old subjects made an average of $2.08 \%$ errors and young subjects averaged $3.12 \%$. This difference was not significant in an analysis of variance; nor were there significant differences between error rates to upper- and lowercase letters. The overall error rates for the letter-matching task were $2.67 \%$ and $3.94 \%$, for old and young subjects, respectively. This difference approached significance $(\mathrm{p}<.10)$ in an analysis of variance, as did the age $x$ session interaction $(p<.10)$. The latter effect reflected a tendency for old subjects to make more errors in Session $2(3.06 \%$ compared with $2.28 \%$ in Session 1), while the young subjects tended to make fewer errors in Session 2 (3.71\% compared with $4.17 \%$ in Session 1 ).

Table 1 presents percentage error rate as a function of trial type, session, and age. As shown, the error rates were highest for the NI trials. Moreover, the frequency of errors for the individual NI letter pairs mirrored, roughly, the response latency data: Errors were most likely for the letters $T$ and $F$ and least likely for the letter $A$. This pattern of errors was shown by both age groups. Young subjects made more DIFF errors than did old subjects during Session 1, but there was no obvious pattern to the distribution of errors across letter combinations for either group. During the second session, when DIFF error rates were more similar for the two groups, the old subjects made more than twice as many errors to the TF combination as they did to the other letter combinations. The young subjects had relatively high error rates for combinations of $A$ and $H$ as well as $T$ and $F$.

Table 1

Percentage Error Rates for the Letter-Matching Task

\begin{tabular}{lrcccccc}
\hline & \multicolumn{3}{c}{ Session 1 } & & \multicolumn{3}{c}{ Session 2 } \\
\cline { 2 - 4 } \cline { 6 - 8 } & PI & NI & DIFF & PI & NI & DIFF \\
\hline Old & .3 & 5.2 & 1.8 & 1.6 & 6.3 & 2.2 \\
Young & 1.0 & 7.0 & 4.3 & 1.8 & 6.5 & 3.3 \\
\hline
\end{tabular}


Finally, error latencies for the PI and NI trials were classified as shorter or longer than the subject's median correct RT for that letter and trial type. One-half to three-fourths of the error trials were relatively fast by this criterion, for both age groups.

\section{Discussion}

The results of the first experiment provide qualified support for the hypothesis that aging is associated with a general reduction in processing speed. For the first three levels of processing complexity (simple, choice, PI), the response latencies of old and young differed by a constant factor, as evidenced by the finding that on a logarithmic scale the difference between age groups did not vary significantly as a function of processing complexity. Moreover, for a subset of the NI pairs (letters $A$ and $H$ ), age differences in response latency could also be attributed to differences in overall processing speed. For other NI pairs (letters $T$ and F), however, the response latencies of the older group were disproportionately long. This latter result suggests that there is an age-related processing deficit, in addition to a general reduction in speed, which affects performance when matching letters on the basis of name. That the deficit is not in the efficiency of letter-name access itself is suggested by the constancy over age of the relative NI-PI difference for certain letters: If advanced age were associated with difficulty in the retrieval of letter names, the response latencies of the elderly subjects should have been disproportionately long for all of the NI letter pairs.

Although the letter-dependent variation was greater for the elderly, both age groups showed a letter effect for NI trials but not for PI trials. This interaction of letter and trial type indicates that there is a stimulus factor that is important for name matches but unimportant for physical matches. The age $\times$ trial type $\times$ letter interaction suggests an age-related difference in sensitivity to this factor.

Several stimulus factors have been advanced as possible contributors to differences in the time required to determine name identity. Posner and Mitchell (1967) suggested "letter frequency" (familiarity), citing the finding that letter-naming time varies inversely with the frequency of the letter in the language (Fitts \& Switzer, 1962). Letter frequency cannot account for the present findings, however, since the letter $T$ is the most frequent letter in the stimulus set (Solso \& King, 1976).

Dainoff and Haber (1970) hypothesized that acoustic similarity affects response latency when comparisons are based on letter names. To test this hypothesis they selected letters from populations of acoustically confusable and nonconfusable let- ters and paired letters within a group. The subjects were instructed to respond on the basis of name identity. As predicted, response latencies were longer and errors were more frequent for confusable than for nonconfusable NI and DIFF pairs, whereas performance for PI pairs did not vary significantly as a function of confusability. However, the letters constituting their confusable and nonconfusable sets, B, D, P, T and F, I, M, Q, respectively, appear to differ in visual as well as acoustic similarity (Dunn-Rankin, 1968; Podgorny \& Garner, 1979; Townsend, 1971a, 1971b). Thus, the effects of "confusability" in this study could have resulted from the visual and/or the acoustic similarity of the letters used.

The possibility that NI response latency varies with visual similarity was raised by Posner (1970). In a simultaneous matching task, subjects were instructed to respond "same" if the letters in a pair were both consonants or both vowels: Latencies for NI pairs were longer for four preselected "difficult" (visually similar) letters $(B, D, G, Q)$ than for four preselected "neutral" letters (F, H, M, R). However, since acoustic similarity was also higher for the difficult letters (except Q), the effect was only tentatively attributed to visual factors.

Although neither the Posner (1970) nor the Dainoff and Haber (1970) study separated the effects of acoustic and visual similarity, they did demonstrate that response latencies for NI pairs vary with letter similarity when acoustic and visual similarity covary. With respect to the present findings, Conrad (1964) found that when people were required to identify letter names spoken against a white-noise background, the names of the letters $A, H, T$, and $F$ were infrequently confused with one another. Phonologically, these four letter names are dissimilar, except that the initial phoneme in the name of the letter $H$ is the name of the letter $A$. Since NI latencies in Experiment 1 were shortest for these two letters, the observed letter effect cannot be attributed to phonological similarity. ${ }^{2}$ Rather,

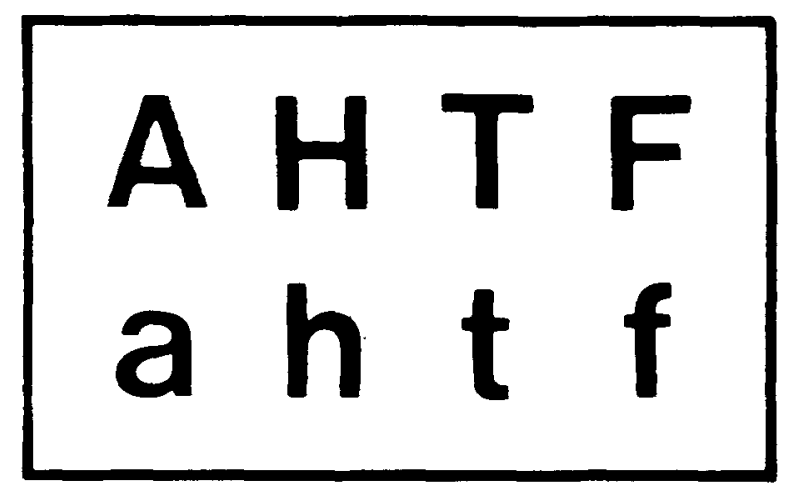

Figure 3. Stimulus letters. 
latencies for the NI pairs appear to have reflected the visual similarity of the letter forms (see Figure 3): Although all of the uppercase forms were somewhat similar (e.g., every uppercase letter consisted of straight lines and contained at least one horizontal line), the uppercase $\mathrm{A}$ was unique in containing diagonal lines and an acute angle. The lowercase $\mathbf{A}$ was also distinctive in that it was shorter and had more curved components than any of the other letter forms. At the other extreme, the lowercase forms of the letters $T$ and $F$ were particularly similar: Their vertical line segments were reflections of each other and their horizontal line segments were identical. (Many subjects, particularly the older ones, commented on their confusability.) In accord with this analysis of letter similarity, name matches were fastest for the letter $A$ and slowest for the letters $T$ and $F$.

\section{EXPERIMENT 2}

Experiment 2 was conducted in order to obtain a measure of the interitem similarities of the letter forms used in the first experiment.

Table 2

Mean Similarity Ratings of Young and Old Adults for Each Letter Combination, Ordered by the Ratings of the Young Adults

\begin{tabular}{ccc}
\hline $\begin{array}{c}\text { Letter } \\
\text { Combination }\end{array}$ & Young & Old \\
\hline tf & 1.25 & 1.59 \\
TF & 2.12 & 3.73 \\
HF & 2.62 & 3.27 \\
AH & 2.69 & 3.50 \\
Tt & 3.12 & 4.05 \\
Ff & 3.37 & 3.41 \\
AF & 3.56 & 5.09 \\
HT & 3.62 & 3.95 \\
ah & 3.81 & 4.45 \\
AT & 4.06 & 5.32 \\
Hh & 4.44 & 3.55 \\
Tf & 4.62 & 3.91 \\
ht & 4.62 & 5.14 \\
hf & 4.69 & 4.73 \\
Ft & 5.00 & 5.09 \\
at & 5.06 & 6.18 \\
af & 5.12 & 6.00 \\
Ah & 5.56 & 5.91 \\
Fh & 5.62 & 5.68 \\
Th & 5.69 & 5.50 \\
Hf & 5.81 & 6.23 \\
At & 5.81 & 6.32 \\
Aa & 6.06 & 5.45 \\
Ht & 6.12 & 5.64 \\
Af & 6.37 & 6.73 \\
Ha & 6.44 & 6.50 \\
Fa & 6.50 & 6.73 \\
Ta & 6.62 & 6.91 \\
\hline
\end{tabular}

Note-1 = extremely similar; 7 = extremely dissimilar. Letter order for a given combination is arbitrary.

\section{Method}

Eleven elderly (mean age $=72.8$ ) and eight young (mean age $=22.9$ ) adults rated the visual similarity of the letter forms presented in Figure 3. The 56 possible permutations of two different forms were reproduced on separate pieces of paper. A 14-cm horizontal line was centered under each pair, with the phrases "extremely similar" and "extremely dissimilar" positioned under the left and right ends of the line, respectively. The subjects indicated the degree of visual similarity of the forms in a pair by placing a mark in the appropriate position on the line. Each subject was given the letter pairs in a different random order, with a copy of Figure 3 on the top of the deck. The subjects were encouraged to examine the stimulus set prior to beginning the rating task and to use the entire range of similarity ratings.

\section{Results and Discussion}

A 7-point, equal-interval scale was used for scoring ( $1=$ extremely similar; $7=$ extremely dissimilar $)$, and the mean rating for each letter combination was determined for each age group. ${ }^{3}$ These data are displayed in Table 2. As predicted, the lowercase forms of the letters $T$ and $F$ were judged as most similar, and decidedly more similar than any other pair of letter forms. The pairs judged as most dissimilar tended to involve the letter $\mathbf{A}$, in one of its forms.

These results indicate that NI response latency in the first experiment varied with the visual similarity of the letter forms. Crist (1981) also found that the time to determine name identity is longer if the stimulus letters are visually similar to other members of the stimulus set.

\section{GENERAL DISCUSSION}

It has been suggested that the elderly are characterized by a specific deficit in the efficiency with which they access highly overlearned information in long-term store, resulting in a positive relationship between age and the magnitude of the difference between NI and PI latencies in a letter-matching task (Hunt, 1978). We proposed an alternative hypothesis, namely, that age-related differences in letter-matching performance reflect differences in overall processing speed.

The present findings do not support the specific deficit hypothesis. However, the hypothesis that aging is associated with a general reduction in processing speed cannot account for the full pattern of results. Rather, a second age-related deficit, in addition to a slower processing speed, was apparent when NI pairs contained letter forms which were visually similar to other members of the stimulus set. Based on the logic of the additive-factors method (Sternberg, 1969), it appears that this second deficit affects the same stage of processing as form similarity. Thus, consideration of the locus of the similarity effect must precede any speculation re- 
garding the nature of the additional age-related processing deficit manifest in Experiment 1.

\section{The Locus of the Similarity Effect}

Although similarity effects in matching tasks are most frequently ascribed to the comparison process (Cohen, 1969; Nickerson, 1972; Thorson, Hockhaus, \& Stanners, 1976), the letter effect for the NI pairs in the present research cannot be accounted for by similarity effects at that stage. If similarity were to affect the comparison of letter names directly, it would be phonological rather than visual similarity and it would affect "different" rather than "same" latencies. On the other hand, if it were assumed that the retrieval and comparison of name codes is not initiated until after the comparison of visual codes, visual similarity could affect NI latency by increasing the time to determine that the letter forms are different and, thus, increasing the time prior to processing the name codes. However, the available data do not support such a sequential model. It appears that the visual and phonological processing systems are relatively independent (Posner, 1978). Moreover, the critical similarity for such a serial process would be between the upper- and lowercase forms of a letter: As shown in Table 2, although the upper- and lowercase forms of the letters $T$ and $F$ were rated as moderately similar, so were the two forms of the letter $H$.

The essence of the interpretation to be advanced here is that the letter effect for the NI pairs reflected differences in letter-identification rather than lettercomparison time. Letter identification is a necessary antecedent to a comparison of letter names (but not to a comparison of letter forms), and it is sensitive to visual similarity (Mayzner, 1972; Shwartz, Pomerantz, \& Egeth, 1977; Rumelhart, Note 1). Logically, at least, identification of a letter requires that the visual form of the letter be judged to be "identical" to a stored internal representation of one of the letters of the stimulus set and/or "different" from the representations of the alternative members of the set. Thus, the critical similarity for letter identification is between the stimulus letter and the other letters in the stimulus set.

Explanations of the association between stimulus similarity and identification performance typically invoke the concept of information accrual. Subjects are thought to accumulate information over time and to respond as soon as the identity of a stimulus can be established with sufficient certainty or, if processing time is restricted, on the basis of partial information (Posner \& Rogers, 1978; Rumelhart, 1977). Thus, the relatively long identification latencies (and/or high error rates) for stimulus forms that are similar to other members of the stimulus set suggest that identification of these forms requires more information, or less rapidly extracted information, than identification of forms that are not similar to other members of the stimulus set.

In the present study, the lowercase forms of the letters $T$ and $F$ had similar general shapes and relatively small distinguishing features. Thus, most analytic (e.g., Estes, 1978; Rumelhart, 1970) and holistic (e.g., Eriksen \& Schultz, 1978; Ginsburg, 1978; Navon, 1977) models of identification could account for the observed letter effect. The disproportionately long latencies shown by the elderly for the NI pairs containing these forms could be explained by postulating a specific age-related deficit in the efficiency with which (certain) higher spatial frequencies or smaller features are processed. However, there are several reasons to question this interpretation. First, although it seems quite tenable that processing speed varies with spatial frequency (or feature size) and that such variation could have contributed to the observed letter effect, the relationship between similarity and performance in discrimination/identification tasks is more general than can be accounted for by this factor: Response latencies reflect similarity when discriminations are based on differences confined to a single physical dimension, such as hue (Bindra, Donderi, \& Nishisato, 1968), and when they are based on differences in the attributes of the referents of symbolic stimuli (Shephard \& Podgorny, 1978). Second, there is no a priori reason to suppose that aging is associated with a disproportionate reduction in the speed with which the small (high spatial frequency) components of a pattern are processed. Indeed, Sekuler, Hutman, and Owsley (1980) found just the opposite for contrast sensitivity: Old and young subjects with good acuity did not differ in their sensitivities to high spatial frequencies, although the elderly were less sensitive (required higher contrast for detection) to low spatial frequencies. Finally, most identification models are based on the implicit assumption that processing mechanisms are errorless. This assumption is almost certainly wrong.

\section{Noise, Aging, and the Similarity Effect}

Although not prevalent in theoretical accounts of identification, the assumption that processing is fallible is central to many models of information processing (e.g., Broadbent, 1971; Tanner \& Swets, 1954; Vickers, 1979). The proponents of such models invoke the concept of "internal noise" and posit that the effects of noise are (on the average) mitigated by additional processing. Several investigators have proposed that increased internal noise is a source of age-related deficits in perceptual tasks (Crossman \& Szafran, 1956; Gregory, 1959; Vickers et al., 1972; Welford, 1965, 1977).

In the interpretation to be advanced here, the 
term "noise" is synonymous with processing error: It is assumed that the nervous system does not transmit information with perfect fidelity and that this error occurs at all levels of the nervous system and at all stages of information processing. Noise level is viewed as the converse of process reliability; the greater the level of noise, the greater the potential variability of processor output given a particular input. Assuming that aging is associated with a general deterioration of nervous system functioning, it is hypothesized that there is an age-related increase in the level of noise.

In the present view, noise is thought to distort the internal representation which results from stimulus processing. To accommodate such distortion plus the physical variability of stimuli with the same identity, a particular label or name (identity) is associated, albeit to different degrees, with a variety of internal representations. Thus, the set of representations to which a particular label is attached can consist of not only the noisy representations of that stimulus object, but also of many of the noisy representations of similar objects; correspondingly, a particular representation will tend to be associated with more than one label, although the strengths of these associations will vary. If the processing of a stimulus results in an internal representation that is associated with only one of the possible stimulus names, that name can be assigned without further processing. If, on the other hand, the internal representation is associated with more than one name, additional processing will be required.

Assuming that the veridicality of an internal representation (at a given point in processing) tends to vary inversely with the level of noise, pattern identification time will be a function of noise level as well as pattern similarity and processing rate: Identification time for forms of sufficiently low similarity should be insensitive to the perturbations of noise, because even extremely distorted internal representations will not be associated with any of the alternative stimulus names. In contrast, the processing needed to identify a form that is similar to other forms in the stimulus set will not only be increased by the presence of noise, but will be a direct function of the level. Therefore, variation of identification time as a function of stimulus similarity should increase with the level of noise.

If, as has been suggested, elderly people suffer from greater internal noise, their identification times should be disproportionately long for confusable letters. The age $\times$ letter interaction for the NI pairs in the present study was of just this form. Since all but one of the DIFF letter combinations contained at least one letter with a form of high visual similarity, and the members of that combination (AH) shared a phoneme, ${ }^{2}$ the letter combination and age $x$ letter combination effects for DIFF should have been smaller than the comparable effects for NI, as was the case. However, the letter-combination effect was significant and there was a tendency, which increased over sessions, for this effect to be greater for the elderly than for the young.

The results of the choice RT task are also consistent with this theoretical position. Since the upperand lowercase letter forms tended to be rated as "dissimilar" (see Table 2), internal noise should rarely have resulted in internal representations for which letter case was ambiguous. Consequently, case identification times should have reflected basic information processing speed, as appears to have been the case.

Neither case discrimination nor letter identification is necessary for a comparison of letter forms. Therefore, the amount of processing needed to determine physical identity is not necessarily affected by the similarity of the letter with respect to the other letters in the stimulus set. However, noise is likely to produce some discrepancy in the representations of the two letters in a PI pair. In a direct extension of the position developed above, we suggest that in order to compare the physical forms of letters the subject evaluates the discrepancy between the internal representations of the two forms (cf. Krueger, 1978). If the discrepancy is less than some criterion, the letters will be judged to be identical; if the discrepancy is greater than this criterion, additional processing will be necessary before a "same" (based on a comparison of either forms or names) or a "different" response can be made with confidence.

If one assumes that differences between representations are evaluated independently of the characteristics of those representations, latencies for PI pairs ought not to vary with letter similarity. A model of this type is consistent with the lack of a letter effect for the PI trials in the present study. However, subjects could set their criteria for identity to reflect the similarity structure of the stimulus set, accepting greater discrepancies for representations with distinctive characteristics. Such a model is suggested by Crist's (1981) finding that letter similarity affected response latency for $P I$ as well as NI pairs in experiments in which subjects were relatively well practiced and onsets of letters in a pair were separated by $35 \mathrm{msec}$.

\section{Internal Noise and Visual Acuity}

We have suggested that the amount of processing necessary to discriminate or identify a stimulus varies with both the level of noise and the similarity of the stimulus, in a nonlinear manner. Since the relationship between stimulus similarity and discrimination latency extends to the limits of the re- 
solving power of the visual system (Vickers et al., 1972), there is a question of the relationship between internal noise and visual acuity. Standard measures of acuity are based on essentially unlimited processing time and are thus a measure of the smallest stimulus for which the asymptote of the speed-accuracy operating characteristic is sufficiently high. Noise, as conceptualized here, determines the rate with which speed is traded for accuracy.

The letters used in the present study were large enough to be accurately identified by all of the subjects: Errors during the letter-matching task were almost invariably followed by a verbal response indicating that the subject was aware of the error. Thus, asymptotic performance for these letters would have been essentially $100 \%$ for all subjects. The question becomes, then, is the rate at which speed is traded for accuracy for a "large" stimulus (i.e., one for which asymptotic performance is very high) a function of the size of the smallest stimulus for which one's asymptotic performance is sufficiently high? The question deserves study.

The relationship between acuity and noise was not examined systematically in the present research. However, a Snellen chart was used to assess the corrected acuities of all but one of the elderly people who participated in the letter-matching experiment. Thirteen of the subjects had corrected binocular acuities of $20 / 30$ or better; the other two had acuities of 20/50. Neither the latency nor the error data of the two subjects with poor acuity were distinctive in any way.

\section{Decision Criteria, Response Latency, and Accuracy}

Implicit in the preceding discussion is the notion that subjects respond on the basis of certain decision criteria. We have been purposely vague on this topic, as a great variety of decision criteria could be postulated (see Vickers, 1979). Although such considerations are beyond the scope of this paper, the possibility that age-related differences in decision criteria contributed to the present findings should be considered. As emphasized by Pachella (1974), criteria differences that result in small differences in accuracy can result in substantial differences in latency, and it has been suggested previously that elderly people are slower because they are more cautious and, therefore, set more stringent response criteria (e.g., Botwinick, Brinley, \& Robbin, 1958).

There was a nonsignificant tendency for the older subjects in the present experiment to be more accurate on both the choice RT and letter-matching tasks. These small differences could have contributed to the overall age differences in latency. However, it is unlikely that differences in speed-accuracy criteria accounted for a sizable proportion of the age effect: The difference between groups in overall error rate on the letter-matching task was $1.89 \%$ in Session 1 and only $.65 \%$ in Session 2 . In spite of this change over sessions in the apparent difference between groups in cautiousness, the age $x$ session interactions in the analyses of the log median latencies for the NI and DIFF trials were not significant $(F<1.0$ in both cases). Moreover, the age $\times$ letter effect for the NI stimuli cannot be accounted for by age differences in cautiousness: The elderly group was not more accurate for the more difficult NI stimuli. Summing over sessions and the letters $T$ and $F$, both the old and young groups made 35 errors $(9.1 \%)$.

\section{Summary}

An attempt was made to distinguish between general and stage-specific age deficits by comparing the performances of young and old adults on a series of tasks (simple RT, choice RT, and letter matching) thought to represent a continuum of processing complexity. Relative differences between young and old were comparable across the first three levels of processing complexity (simple, choice, PI) and a subset of the NI pairs. This invariance across levels of complexity is most readily interpreted in terms of a general age-related reduction in processing speed. Evidence that aging is associated with a second processing deficit was provided by disproportionate age differences for another subset of the NI pairs. The pairs that resulted in larger age differences were shown to be those containing letter forms which were visually similar to other members of the stimulus set. It is suggested that the disproportionately long latencies of the elderly resulted from an age-related increase in internal noise which affects discrimination/identification times for confusable stimuli.

\section{REFTRENCE NOTE}

1. Rumelhart, D. A. A multicomponent theory of confusion among briefly exposed alphabetic characters (Tech. Rep. 22). University of California, San Diego, Center for Human Information Processing, November 1971.

\section{RGFERENCES}

Anders, T. R., Fozard, J. L., \& Lillyquist, T. D. The effects of age upon retrieval from short-term memory. Developmental Psychology, 1972, 6, 214-217.

Bindra, D., Donderi, D. C., \& Nishisato, S. Decision latencies of "same" and "different" judgments. Perception \& Psychophysics, 1968, 3, 121:130.

Birren, J. E. Age changes in speed of behavior: Its central nature and physiological correlates. In A. T. Welford \& J. E. Birren (Eds.), Behavior, aging and the nervous system. Springfield, Ill: Thomas, 1965 .

Birren, J. E. Psychophysiology and speed of response. American Psychologist, 1974, 29, 808-815. 
Botwinicx, J. Aging and behavior: A comprehensive integration of research findings. New York: Springer, 1973.

Botwinick, J., Brinley, J. F., \& RoBbin, J. The interaction effects of perceptual difficulty and stimulus exposure time on age differences in speed and accuracy of response. Gerontologia, 1958, 2, 1-10.

Brondbent, D. E. Decision and stress. New York: Academic Press, 1971.

Chinas, A., \& Atkinson, R. C. Individual differences and interrelationships among a select set of cognitive skills. Memory \& Cognition, 1976, 4, 661-672.

Cohen, G. Some evidence for parallel comparisons in a letter recognition task. Quarterly Journal of Experimental Psy. chology, 1969, 21, 272-279.

Conrad, $\mathbf{R}$. Acoustic confusions in immediate memory. British Journal of Psychology, 1964, 55, 75-84.

Crist, W. B. Matching performance and the similarity structure of the stimulus set. Journal of Experimental Psychology: General, 1981, 111, 269-296.

Crossman, E. R. F. W., \& Szafran, J. Changes with age in the speed of information intake and discrimination. Experientia Supplementum, 1956, 4, 128-135.

Dainhorf, M. J., \& Haber, R. N. Effects of acoustic confusability on levels of information processing. Canadian Journal of Psychology, 1970, 24, 98-108.

Dunn-Rankin, P. The similarity of lower case letters of the alphabet. Journal of Verbal Learning and Verbal Behavior, $1968,7,990-995$.

Erik8en, C. W., \& Schultz, D. W. Temporal factors in visual information processing: A tutorial review. In J. Requin (Ed.), Attention and performance VII. Hillsdale, N.J: Erlbaum, 1978.

Estzs, W. K. Perceptual processing in letter recognition and reading. In E. C. Carterette \& M. P. Friedman (Eds.), Handbook of perception (Vol. 9), New York: Academic Press, 1978.

Fitrs, P. M., \& Switzen, G. Cognitive aspects of information processing: I. The familiarity of S-R sets and subsets. Journal of Experimental Psychology, 1962, 63, 321-328.

GinsBurg, A. P. Visual information processing based on spatial filters constrained by biological data (AMRL-TR-78-129, Vols. 1 and 2). Wright-Patterson Air Force Base, Ohio: Aerospace Medical Research Laboratory, December 1978. (NTIS No. AD-A090 117/3)

GregoRY, R. L. "Neurological noise" as a factor in aging. Proceedings of the Fourth Congress of the International Association of Gerontology, 1959, 1, 314-324.

Hunt, E. Mechanics of verbal ability. Psychological Review, 1978, 85, 109-130.

Hunt, E., Frost, N., \& Lunnebora, C. Individual differences in cognition: A new approach to intelligence. In $\mathbf{G}$. Bower (Ed.), Advances in learning and motivation (Vol. 7). New York: Academic Press, 1973.

Hunt, E., Lunnebora, C., \& Lewis, J. What does it mean to be high verbal? Cognitive Psychology, 1975, 7, 194-227.

HunT, E., \& MAcLeod, C. M. The sentence-verification paradigm: A case study of two conflicting approaches to individual differences. Intelligence, 1978, 2, 129-144.

Kentina, D. P., \& Bobeitr, B. Individual and developmental differences in cognitive processing components of mental ability. Child Development, 1978, 49, 155-167.

Krueoer, L. E. A theory of perceptual matching. Psychological Review, 1978, 85, 278-304.

MAYZNER, M. S. Visual information processing of alphabetic inputs. Psychonomic Monograph Supplements, 1972, 4(13, Whole No. 61), 239-243.

Navon, D. Forest before trees: The precedence of global features in visual perception. Cognitive Psychology, 1977, 9, 353-383.

Nickenson, R. S. Binary-classification reaction time: A review of some studies of human information processing capabilities. Psychonomic Monograph Supplements, 1972, 4(17, Whole No. 65).
Pachella, R. G. The interpretation of reaction time in information-processing research. In B. H. Kantowitz (Ed.), Human information processing: Tutorials in performance and cognition. Potomac, Md: Erlbaum, 1974.

Podoorny, P., \& Garner, W. R. Reaction time as a measure of inter- and intraobject visual similarity: Letters of the alphabet. Perception \& Psychophysics, 1979, 26, 37-52.

Posnen, M. I. On the relationship between letter names and superordinate categories. Quarterly Journal of Experimental Psychology, 1970, 22, 279-287.

Posner, M. I. Chronometric explorations of mind. Hillsdale, N.J: Erlbaum, 1978.

Posmer, M. I., \& Mitchell, R. F. Chronometric analysis of classification. Psychological Review, 1967, 74, 392-409.

Posner, M. I., \& Rogers, M. G. K. Chronometric analysis of abstraction and recognition. In W. K. Estes (Ed.), Handbook of learning and cognitive processes (Vol 5): Human information processing. Hillsdale, N.J: Erlbaum, 1978.

RUMElHART, D. E. A multicomponent theory of the perception of briefly exposed visual displays. Journal of Mathematical Psychology, 1970, 7, 191-218.

RUMELHART, D. E. Introduction to human information processing. New York: Wiley, 1977.

Sexular, R., Hutman, L. P., \& Owsley, C. J. Human aging and spatial vision. Science, $1980,209,1255-1256$

She Phard, R. N., \& Podgonny, P. Cognitive processes that resemble perceptual processes. In W. K. Estes (Ed.), Handbook of learning and cognitive processes (Vol. 5): Human information processing. Hillsdale, N.J: Erlbaum, 1978.

Shwartz, S. P., Pomerantz, J. R., \& Eoeth, H. E. State and process limitations in information processing: An additive factor analysis. Journal of Experimental Psychology: Human Perception and Performance, 1977, 3, 402-410.

Solso, R. L., \& King, J. F. Frequency and versatility of letters in the English language. Behavior Research Methods a Instrumentation, 1976, 8, 283-286.

Sternberg, S. The discovery of processing stages: Extensions of Donder's method. Acta Psychologica, 1969, 30, 276-315.

Tanner, W. P., \& Swets, J. P. A decision-making theory of visual detection. Psychological Review, 1954, 61, 401-409.

Thonson, G., Hockhaus, L., \& Stanners, R. F. Temporal changes in visual and acoustic codes in a letter-matching task. Perception \& Psychophysics, 1976, 19, 346-348.

Townsend, J. T. Alphabetic confusion: A test of models for individuals. Perception \& Psychophysics, 1971, 9, 449-454. (a)

Townsend, J. T. Theoretical analysis of an alphabetic confusion matrix. Perception \& Psychophysics, 1971, 9, 40-50. (b)

VICKe Rs, D. Decision processes in visual perception. New York: Academic Press, 1979.

Vickers, D., NeTtelbeck, T., \& Willson, R. J. Perceptual indices of performance: The measurement of "inspection time" and "noise" in the visual system. Perception, 1972, 1, 263-295.

WALsh, D. A. Age differences in central perceptual processing: A dichoptic backward masking investigation. Journal of Gerontology, 1976, 31, 178-185.

Welford, A. T. Performance, biological mechanisms and age: A theoretical sketch. In A. T. Welford \& J. E. Birren (Eds.), Behavior, aging and the nervous system. Springfield, Ill: Thomas, 1965.

WELfORD, A. T. Motor performance. In J. E. Birren \& K. W. Schaie (Eds.), Handbook of the psychology of aging. New York: Van Nostrand Reinhold, 1977.

\section{NOTES}

1. The proportionality of treatment effects could also be assessed by comparisons of individual performance ratios. An analysis of variance conducted on the quotients obtained by dividing the median response latencies representing a subject's performance on the letter-matching task by his or her mean me- 
dian latency on the simple RT task gave essentially the same overall picture as was given by the log millisecond analysis.

2. For the DIFF pairs, latencies to the AH combination were relatively long (second only to the TF combination), suggesting that phonological similarity did affect the time required to determine that two letter forms did not share the same name.

3. Although the average similarity ratings of the old and young groups did not differ substantially for any letter pair, there was considerable variability among raters for letter pairs with moderate average similarities. Moreover, disparate ratings for the two permutations of a pair were not uncommon for subjects in the elderly group: $14.7 \%$ of the elderly group's ratings differed by at least three points, compared with $3.6 \%$ of the young group's. Thus, if one assumes that similarity judgments reflect distances in a common multidimensional perceptual space, there was substantial intersubject and, at least for the elderly, intrasubject variation in the relative salience or importance of the underlying dimensions.

(Manuscript received July 13, 1982; revision accepted for publication December 6, 1982.) 